\title{
Strategies for community-based dementia education and creating sustainable change: Lessons learned from the Community Outreach Education Program
}

\author{
Cathleen M Connell, PhD \\ Benjamin Walter, MPH \\ Margaret La Pietra Kunz, MPH \\ Sara B. Holmes, MPH
}

\begin{abstract}
The Community Outreach Education Program (COEP) was established to disseminate information about the diagnosis, assessment, management, and treatment of dementia to health care professionals, service providers, staff of community organizations and voluntary agencies, and family caregivers in communities throughout Michigan. This paper will describe the activities implemented as part of outreach educational interventions in Bay City, Saginaw, Grand Rapids, and Kalamazoo, Michigan. Lessons learned about community-based dementia education and strategies for creating sustainable change are discussed.
\end{abstract}

\section{Introduction}

The Community Outreach Education Program (COEP) was established to disseminate information about the diagnosis, assessment, management, and treatment of

Cathleen M Connell, PhD, Director, Education and Information Transfer Core, Michigan Alzheimer's Disease Research Center, University of Michigan, Ann Arbor, Michigan.

Benjamin Walter, MPH, Health Educator, Michigan Alzheimer's Disease Research Center, University of Michigan, Ann Arbor, Michigan.

Margaret La Pietra Kunz, MPH, Health Educator, Michigan Alzheimer's Disease Research Center, University of Michigan, Ann Arbor, Michigan.

Sara B. Holmes, MPH, Coordinator, Education and Information Transfer Core, Michigan Alzheimer's Disease Research Center, University of Michigan, Ann Arbor, Michigan.
Alzheimer's disease (AD) and other dementing illnesses. In contrast to typical continuing education activities in which the goals and target audience are narrowly defined, the COEP seeks to involve a broad range of health care professionals, service providers, and family caregivers in programs that address locally-defined needs and concerns. The long term objective of the COEP is to create a sustainable impact on the community by fostering a knowledgeable service delivery network with the capacity to respond appropriately to the needs of people with dementing illnesses and their families.

This paper will describe the activities implemented as part of outreach educational interventions in four communities in Michigan-Bay City, Saginaw, Grand Rapids, and Kalamazoo. Lessons learned and strategies for creating sustainable change are examined. Interventions were conducted in these sites since 1996 as part of a six-year funding period. Previous reports have summarized the planning and community assessment phases of the project ${ }^{1,2}$ and described a process evaluation of interventions implemented in six communities between 1992 and $1995 .^{3}$

\section{Overview of the COEP process}

In each of the 10 selected intervention sites in Michigan, a local advisory board of health care professionals, dementia service providers, and representatives of community, government, and voluntary agencies which address the needs of people with dementia was established. The advisory boards provided local leadership, coordinated 
publicity, made local arrangements for the educational interventions, and contributed to the community assessment and design, implementation, and evaluation phases of the project. Two full-time Master's trained Health Educators employed by the COEP coordinated the activities of the advisory boards.

In collaboration with the local advisory boards, COEP staff conducted a dementia-specific community assessment in order to identify community resources and strengths, gaps in service availability, and strategies to increase coordination and communication among service providers. In Bay City and Saginaw, data were collected via focus group and in-depth personal interviews with service providers, physicians, and family caregivers. For the other two sites, an abbreviated needs assessment was conducted via focus group interviews with board members and caregiver support groups.

Based on the community assessment data, priorities for the intervention were established as a collaborative process between COEP staff and the advisory boards in each site. To address the identified priorities, COEP staff worked monthly with the advisory boards in each site for approximately one year, meeting on a monthly basis. The COEP reflects the fundamentals of community health education by listening and responding to the community's assessment of its strengths and needs, recognizing and building on community strengths, and fostering a high level of community participation. ${ }^{4}$ In the next section, activities implemented as part of the educational interventions in the four communities are described in chronological order.

\section{Bay City}

A group of dementia-related service providers referred to as the Bay County Dementia Round Table had been meeting monthly for two years prior to involvement with the COEP. This group agreed to serve as the advisory board for the COEP activities in Bay City. Representatives from the majority of dementia-related service agencies in the area participated in the Round Table-including the local hospital, non-profit community organizations, an adult day care center, and several private nursing homes. In addition, several caregivers participated in the group. During the COEP's tenure, group members included nearly 40 people, with approximately 12 to 20 attending each meeting.

COEP staff conducted 23 interviews with key informants representing the local dementia service-delivery system in Bay County. Names of the key informants were solicited from members of the Dementia Round Table. In addition, one focus group interview with physicians and two focus group interviews with caregivers were conducted. Interview questions addressed the coordination of and gaps in dementia services; educational needs of physicians, service providers, family caregivers, and the general public as well as strategies for improving services and providing education.

Based on the interview data, a preliminary report was developed to inform the process of planning the intervention. The top priority needs identified in the report included caregiver education and support, general public education, and physician education. By consensus, the Round Table chose the following three activities to address these needs:

- Complete steps necessary to launch a local dementia resource center;

- Increase public awareness of the warning signs of dementia and where to seek help by developing and distributing educational materials; and

- Provide educational outreach to physicians, including offering training sessions to office staff and developing materials for use in physician offices.

The COEP's arrival in the community provided incentive to address the educational and referral needs of the general public by helping the members of the Round Table complete the Bay County Dementia Resource Center and information line. Work on this effort had begun prior to the COEP's involvement in Bay City. The Resource Center and information line was launched in November 1996, as part of a community-wide open house that attracted over 100 community members. This new service offers a local phone number and resource center that people can call or visit to receive information about dementia and local community services. Community members can visit the center to look at materials, watch videos, and check out books. The information line is staffed during business hours by the program director at the adult day care center where it is located.

Several educational materials were developed to publicize the Bay County Dementia Resource Center and information line, increase knowledge of the warning signs of dementia, and help to decrease the stigma of having a family member with dementia. These materials, including wallet and Rolodex cards, as well as posters, were designed collaboratively by the Round Table and COEP staff. Wallet cards were printed with the heading, "Alzheimer's is a disease, not a disgrace." The cards include the phone number for the Resource Center and information line and the warning signs of dementia. 
Wallet cards were distributed to caregivers and the general public via support groups, physician offices, and dementia service providers. About 4,000 cards have been distributed in the Bay City area. Wallet cards were also designed for caregivers to use when they are in public with their care receiver. The card says, "Please be patient. The person with me has Alzheimer's disease. Thank you for understanding." The card allows caregivers to inform others about the reason for unusual behaviors while avoiding the potential embarrassment or discomfort that could be caused by a verbal explanation. The cards have been well received by caregivers, with 500 copies distributed in the community. Three hundred 11 " by 17" posters were printed for display in physician offices and health and human service agencies. The poster includes the warning signs of dementia and the telephone number for the information line. A pocket that holds the information line wallet cards is attached to the poster. Finally, 500 Rolodex cards with the Resource Center and information line phone number, address, hours of operation, and an abbreviated list of services were distributed to professional providers.

To address physician outreach, COEP staff organized a Grand Rounds presentation on dementia recognition, assessment, and treatment at a local hospital. Only 13 physicians attended the session, which was offered by a neurologist with expertise in dementing illnesses. In response to this relatively low turnout and previous challenges experienced by members of the Round Table in reaching local physicians, the group decided to offer dementia training to physician office staff (e.g., nurses, medical assistants, receptionists).

A training session and materials, referred to as "Dementia: Recognition, Assessment, and Referral" were developed by a work group of Round Table members and COEP staff. The objectives of the training were to increase knowledge and awareness of:

- Dementia and dementing illnesses;

- The importance of early diagnosis and assessment;

- Specific strategies for diagnosis and assessment; and

- Appropriate referrals to community-based services.

Invitations to the training session were sent to 90 physician offices in the Bay City area. Fifteen office staff members, a physician, and a neuropsychologist attended. At a one month follow up, COEP staff completed telephone interviews with the six participating physician offices to evaluate the training program. Results indicated that although most participants reported that they were more alert to warning signs when interacting with patients, only one used the recommended assessment tools. None of the office staff discussed the training session with their physician employers, although most of the participating offices displayed the memory loss poster and wallet cards. Participants reported feeling uncomfortable suggesting changes in office procedures and a lack of opportunity and time to implement the training guidelines and recommendations. Because attendance at this program was low and the outcomes were disappointing, physician outreach continues to be a priority in this area.

In the 18 months since the completion of the intervention in Bay City, members of the Round Table continue to develop the Bay City Dementia Resource Center and information line by collecting and disseminating new materials. Members have also distributed the educational materials developed as part of the intervention, including the posters and wallet cards. Between eight and 12 Round Table members continue to meet bi-monthly, serving primarily in an advisory and advocacy capacity.

\section{Saginaw}

Unlike Bay City, there was no existing dementiarelated advisory group for the COEP to join in Saginaw. The director of a county agency that addresses the needs of older adults volunteered to draft a list of local professionals concerned with dementia to invite to an initial orientation meeting. Seventy-two people were invited, representing the entire range of dementia care services in Saginaw, from information and referral agencies to nursing homes. Of the 33 people who attended the meeting, 22 volunteered to serve on the newly created Saginaw County Advisory Board. Participating agencies included the local hospital systems, home health agencies, caregivers, and a representative of the local chapter of the Alzheimer's Association. Over the course of the year, between 12 and 22 people attended monthly meetings. By the end of the intervention period, there were 60 individuals on the advisory board's mailing list, representing over 30 organizations.

As part of the community assessment, COEP staff conducted 26 key informant interviews with local dementia service providers and caregivers using a modified interview guide that was developed for use in Bay City. Two focus group interviews with caregivers were conducted, and information was collected from nine physicians via interview or questionnaire. As part of several initial meetings, members of the Saginaw County Advisory Board established three goals: 
- To facilitate networking among members;

- To increase knowledge and awareness of dementia; and

- To conduct educational outreach to caregivers, the general public, and physicians.

By consensus, three activities were chosen to address these needs: a dementia services resource directory; an educational program for caregivers; and a general public education campaign.

The first activity completed in this site was the 68page Saginaw County Dementia Services Resource Directory, which includes detailed information about over 80 local service providers. The directory includes information designed to help make comparisons among available services and educational information that can be copied and disseminated to caregivers. Three hundred copies were distributed by advisory board members.

Next, the advisory board and COEP staff worked collaboratively to plan the caregiver education series, consisting of six two-hour weekly sessions at a local hospital. The sessions were offered on Saturdays as a way to increase attendance. Topics addressed in the series were based on the results of a survey of local caregivers and included community services, managing difficult behaviors, medications and current research, activity planning, coping and caregiver stress, communicating with people with dementia, and financial and legal planning. Presenters included advisory board members and local dementia experts. Approximately 140 people attended at least one class, 70 percent of whom were caregivers.

Efforts to target the general public included the development and distribution of educational outreach materials and the development of the Mid-Michigan Dementia Speakers Bureau. Outreach materials that were first developed in Bay City were shared with the Saginaw group. Advisory board members decided to modify these same materials, including the wallet card, the "Please Be Patient" card, the memory loss poster, and the Rolodex card.

The purpose of the Mid-Michigan Dementia Speakers Bureau is to increase knowledge and understanding of dementia among the general public, professional service providers, and caregivers. The Speakers Bureau consists of eleven individuals recruited from the Bay County Round Table and the Saginaw County Advisory Board, including nurses, social workers, and representatives of several local dementia service agencies. The training for the Speakers Bureau was offered during three separate 2$1 / 2$ hour sessions and focused on an overview of dementia and caregiving issues. Participants helped design the materials and were then given the opportunity to master the information and practice giving a presentation. Volunteers who completed the training agreed to provide at least three presentations per year and train other volunteers interested in joining the Speakers Bureau.

To address physician education, COEP staff arranged for a neurologist to give a presentation to physicians on dementia detection and diagnosis during Grand Rounds at a local hospital. Approximately 28 people attended. In addition, an outreach program was developed to disseminate educational materials and information to physicians. As part of the program, materials developed by COEP staff were delivered by home health agency representatives to physician offices. Eleven physicians volunteered to serve as program consultants and review materials prior to distribution. Packets of educational materials on dementia and dementing illnesses were delivered to 20 physician offices in the first year of operation.

Since the end of the COEP intervention period in Saginaw, approximately 10 to 12 advisory board members have continued to meet to plan community outreach activities. For example, a local service agency for older adults developed a dementia resource center at its facility. The advisory board also held a conference on elder abuse and repeated the caregiver education series.

\section{Grand Rapids}

Members of the West Michigan Dementia Consortium had been meeting for several months prior to the COEP's involvement in the Grand Rapids area. This group originally convened in response to the disaffiliation of the local chapter of the Alzheimer's Association with the goal of maintaining support services for family and professional caregivers. At the beginning of the COEP intervention, the Consortium included 13 representatives of the local dementia service delivery network. Several physicians and representatives of home health agencies and hospitals were invited to join the group. Over the course of the intervention period, meeting size ranged from eight to 12 people.

Rather than conducting a formal community assessment involving key informant and focus group interviews, the Consortium decided to identify local needs during the group's meetings. As part of this process, members generated separate lists of the services that they felt should be available for people with dementia and names of local agencies to provide those services. As a result, members were able to identify the unavailable services in their community.

Consortium members determined that the most pressing unmet needs included affordable respite care, care management services, and physician education. The Consortium decided by consensus to focus its efforts exclusively on 
improving physician care of people with dementia and their families. This decision was made for two reasons. First, no local agency was currently providing physician education. Second, addressing physician education was perceived as taking best advantage of the resources available to the COEP, particularly the expertise of the neurologists at the Michigan Alzheimer's Disease Research Center at the University of Michigan. By their outreach efforts to physicians, Consortium members hoped to increase:

- The frequency and accuracy of dementia recognition, assessment, and diagnosis;

- Physician referral to appropriate community services; and

- Opportunities for patient and caregiver education at the physician's office.

To accomplish these goals, the Consortium selected three strategies:

- Group presentations to physicians;

- Visits to physician offices by representatives of home health agencies; and

- Dissemination of educational materials to caregivers.

Next, Consortium members recruited 10 physicians and two psychologists to serve as consultants to review program materials.

To inform their efforts, Consortium members conducted a survey of primary care physicians in Kent County to assess their level of interest in specific topics (e.g., assessment and diagnosis, community services) and preferred format(s) for receiving dementia-related information (e.g., written materials, videos). The survey was sent to 453 primary care physicians in Kent County. Only 37 surveys were completed and returned. A second mailing resulted in the return of an additional $76 \mathrm{com}-$ pleted surveys, for a total of 113 postcards (and a 25 percent return rate). Results indicated that physicians were most interested in assessment and diagnosis, followed by community services, medical management, caregiver education, and current research. Respondents indicated that they most preferred to receive information in the form of written materials, followed by a CME presentation, a presentation at the physician's office, a video, and a CME course offered on the internet.

Despite repeated attempts, Consortium members were unable to schedule group presentations for physicians at local hospitals. Consortium members were successful, however, in recruiting about 25 physicians to attend a presentation about dementia at a local hotel that was planned in collaboration with representatives of the local offices of two large pharmaceutical companies.

The Consortium invited home health agencies to disseminate information and materials to physicians' offices, following the model used in Saginaw. COEP staff gathered appropriate materials to address the needs identified by the physician survey, particularly guidelines for the diagnosis and assessment of dementia. Although gaining access to physicians to introduce the program was difficult, four home health agencies are distributing materials to approximately 20 physician offices. In response to a question included in the survey, an additional 34 local physicians expressed an interest in having a Consortium member come to their office to discuss issues related to the care of their patients with dementia.

The posters and wallet and Rolodex cards developed for use in Saginaw and Bay City were also used in Grand Rapids. In addition, prescription pads with the Alzheimer's Association toll free number, description of services, and place for the physician's signature were developed. These pads were designed to capitalize on the authority physicians often have in motivating patients and families to seek additional services. Table tents with the Alzheimer's Association (AA) number, list of potential dementia symptoms, and tips on how to enhance communication between patient and physician were also developed. The tents were distributed to hospitals and other community sites by Consortium members. COEP staff also produced a physician appointment reminder form which provides space for caregivers to list changes they have noticed in their family member with dementia since the last doctor's visit. The form also includes suggestions for improving communication with the physician, a space for questions and concerns, and information on the diagnosis of dementia. These forms are being distributed to caregivers by agencies represented by the Consortium, home health agencies and participating physicians.

At the conclusion of the COEP intervention, the Consortium decided by consensus to continue their efforts to educate local physicians for the following year. The West Michigan chapter of the Alzheimer's Association, which began to serve the area after the dissolution of the Grand Rapids chapter, agreed to coordinate the physician office visits by home health agency representatives.

\section{Kalamazoo}

To recruit participants for the COEP intervention, the local chapter of the Alzheimer's Association invited 23 representatives of the local dementia service delivery 
network to an initial meeting. Sixteen volunteers attended, all of whom agreed to serve on a COEP advisory board referred to as the Kalamazoo County Dementia Consortium. During the COEP's tenure, the Consortium grew to include 37 people, and meeting size ranged between eight and 15 people.

The Consortium followed the same planning process used in Grand Rapids. Specifically, members generated a list of dementia services that were currently available in their community. Next, Consortium members and other local dementia experts determined the most pressing dementia related needs, including respite care, diagnostic and assessment centers, case management, end stage placement, transportation, and information and referral services. Target audiences for dementia education included service providers, caregivers, and staff of home health agencies and nursing homes.

By consensus, the group decided to focus on two toppriority needs - enhancing information and referral services-and increasing access to respite and in home services. To improve appropriate and timely use of such services, the group decided to:

- Increase knowledge and awareness of dementia services among professional and family caregivers;

- Increase knowledge of the warning signs of dementia among the general public;

- Increase providers' skills in assessing the service needs of patients and families; and

- Enhance the information and referral infrastructure.

To accomplish these objectives, specific activities planned for Kalamazoo included the development of a dementia resource directory, a web site, and the dissemination of educational materials. Three hundred copies of a 100-page resource directory of dementia services in Kalamazoo County were distributed to professional service providers. The directory was patterned after the one developed in Saginaw. A web site was established to provide another means of educational outreach to family and professional caregivers. The site includes information found in the resource directory and provides links to other dementia-related sites. Several of the educational materials originally developed by COEP staff and the Bay County Dementia Round Table were modified for use in Kalamazoo, including the warning signs poster, wallet and Rolodex cards, and prescription pads.

To increase knowledge and awareness of dementia among professional and family caregivers, the Consortium conducted a train-the-trainer program. Thirty-five providers from home health agencies, assisted living facilities, senior centers, volunteer organizations, and a nurse parish network attended the consortium training. This training was entitled Helping People with Alzheimer's Disease: A Train-the-Trainer Program. The one-day program was presented by a local consultant and taught participants how to disseminate the curriculum to interested audiences. Topics addressed included the basics of dementia, caregiving issues, techniques for teaching adult learners, communicating with people with dementia, understanding difficult behaviors, and community resources.

Since the end of the COEP intervention period, the Consortium continues to meet bi-monthly. The local Alzheimer's Association and the Area Agency on Aging facilitate and coordinate the meetings. Consortium members are creating a database from the resource directory which will make it easier to update information and conduct an electronic search for specific services. The local chapter of the Alzheimer's Association agreed to assume responsibility for the directory database. Consortium members volunteered to update the web site and post current events, announcements, and minutes from Consortium meetings. To assure the continuation of the train-the-trainer program, COEP staff provided Consortium members with a plan for maintaining contact with the program participants.

\section{Implications for practitioners}

Several implications can be drawn from the process of planning and implementing the interventions in each of the four sites. A community assessment is an essential first step in identifying local needs and resources. In the first two sites (Bay City and Saginaw), in-depth interviews with a broad representation of the dementia service delivery network were conducted as part of a comprehensive community assessment. Although the information gathered was valuable in providing locallyrelevant information about the dementia service delivery network and recruiting members of the advisory boards, the process was very labor intensive and was perceived by the advisory boards as delaying the time available for program planning and implementation. In Kalamazoo and Grand Rapids, the local advisory boards decided to conduct a less comprehensive and time consuming community assessment that relied heavily on input from their members and the experiences of COEP staff in other intervention sites. Although the abbreviated format for the community assessment yielded valuable information, it did not directly reflect the input of all of the possible stakeholders in the intervention (e.g., physicians, caregivers). 
Members of local advisory boards should weigh the pros and cons of various formats and types of assessments before selecting one that is best targeted to their needs and can be used to inform follow up activities after the official project period ends.

As discussed by Israel et al., ${ }^{5}$ the effectiveness of interpersonal relationships established between community residents and the project staff is key to successful planned change efforts. As with the previous six sites, ${ }^{3}$ COEP staff found it valuable to address group process issues with the advisory boards early in the planning process. For example, considerable effort was directed to deciding how group decisions would be made because efforts to facilitate member influence foster satisfaction and commitment among participants. ${ }^{6}$ In most cases, consensus decision making was found to be an effective strategy, marked by a structured process, time for questions and discussion, and the opportunity for disagreement. Variations on the nominal group technique, in which members vote individually and then discuss the results before coming to a final decision also worked well. Attention to group process issues was especially important in those sites where an existing committee or group could not be identified to serve as an advisory board (i.e., Saginaw, Kalamazoo). In these sites, discussion addressing the expectations and roles of advisory board members was particularly important. ${ }^{7}$

In each of the four sites, attendance and participation in advisory board meetings was not consistent, with multiple changes in membership and leadership. The resulting variability in group dynamics decreased ownership of the project and complicated the process of making decisions by consensus. To respond to the realities of working with unpaid volunteers with demanding professional responsibilities and schedules, meeting times and locations were set by the advisory boards and members were encouraged to provide feedback by mail. Both attendance and participation are facilitated when participants have input into the location and scheduling of meetings. ${ }^{7}$

Several other strategies to keep advisory members satisfied with their involvement in the COEP were implemented. For example, COEP staff attempted to create a cohesive, task-oriented, and innovative environment for all advisory board meetings. ${ }^{6}$ In addition, advisory board members were encouraged to participate fully in the COEP by organizing activities and working toward goals outside of scheduled meetings. ${ }^{6}$ When effective, these strategies are thought to decrease perceived costs and increase perceived benefits of participation, produce members who were more satisfied and involved in the process, and maintain membership over time. ${ }^{6}$

After the community assessment data were shared with the advisory boards, each site established specific educational objectives for their particular local intervention. Across the four sites, the objectives were similar. For example, increasing community wide knowledge and awareness of dementia and educational outreach to caregivers, the general public, and physicians were selected in three of the four sites (i.e., Bay City, Saginaw, and Kalamazoo). In Grand Rapids, the advisory board decided to focus the intervention exclusively on physician education. In Saginaw, one of the objectives of the intervention was to facilitate networking among advisory board members. In Kalamazoo, activities were designed to enhance local information and referral services available to professional providers.

Several activities designed to address the objectives in each community were particularly effective. First developed in collaboration with the advisory board in Bay City, educational materials (e.g., posters, wallet cards) were used to disseminate information about dementia to various target audiences. These materials were so well received in Bay City that they were revised for distribution in Saginaw, Grand Rapids, and Kalamazoo. Local pharmaceutical companies were very generous in contributing funds for the development and production of these materials. Although it is difficult to assess the effectiveness of materials that are disseminated at the community level, the local chapters of the Alzheimer's Association that serve the COEP intervention sites reported that approximately 5 to 10 percent of their helpline calls were prompted by these materials.

In Bay City, the advisory board decided to establish a dementia resource center, filling a gap in the local service delivery network. Planning a community wide open house was an effective way to introduce caregivers, service providers, and community leaders to this new facility. The resource directories developed in Saginaw and Kalamazoo were distributed to a wide range of health professionals and local service providers, who are now able to respond more appropriately to the needs of people with dementia and their families. For example, it is now possible for physicians to refer to their directory and share current information about respite programs, longterm care facilities, or lawyers specializing in elder law with family caregivers. In addition to creating a useful end product, the process of developing the directories served to increase the advisory board's awareness of the local service delivery system.

An educational program based on a train-the-trainer model was implemented in Kalamazoo. By design, this type of program offers great potential for a sustainable impact because participants gain the skills required to disseminate information about dementia. A major challenge of implementing a train-the-trainer program, however, is the difficulty of devoting sufficient time to both a 
rigorous educational curriculum and sufficient skillbuilding exercises so that participants can serve effectively in their role as trainers. In addition, the evaluation of such programs is complex, given the fact that they are ongoing and serve multiple target audiences (i.e., trainers, participants, trainees).

A caregiver education program was implemented in Saginaw. Program success was attributed, in part, to the decision to recruit local service providers to contribute to the curriculum and serve as presenters. These providers can revise and repeat the program as needed without reliance on COEP staff and make contacts with new caregivers to sustain the program.

Because the interventions designed and implemented in each of the four sites had multiple components and intended target audiences, efforts to plan a comprehensive outcome evaluation have been challenging. While the impact of the COEP on overall dementia care practices and access to available services may be the ideal indicators of program success, evaluation of these outcomes is rarely possible as part of a community-based outreach initiative. ${ }^{8}$ In the case of the COEP, funding and personnel resources were extremely limited, resulting in compromises to a rigorous outcome evaluation. More importantly, the advisory boards demonstrated a strong preference for channeling COEP resources to program planning and implementation and resisted efforts to devote time and energy to evaluation efforts that were, at times, viewed primarily as relevant only to a research agenda. Finally, the dynamic nature of change over time in the contexts related to potential outcomes further complicated the efforts of COEP staff to assess program impact. For example, the disaffiliation of the local chapter of the Alzheimer's Association in Grand Rapids created a temporary gap in information and referral services related to dementia prior to the arrival of the COEP in this site. This gap complicated efforts to assess the impact of physician outreach activities designed to increase referral to appropriate community services. Given all of these factors, COEP staff focused their efforts on process rather than outcome evaluation. Process evaluation complements outcome evaluation by providing data that describes how a program was implemented, to whom services were delivered, how participants and sites responded to the intervention, and the extent to which the target population was reached. ${ }^{5,9}$

Because families often first turn to their primary care physician when a loved one exhibits symptoms of dementia, physicians represent an important target audience for educational efforts. Outreach programs that target physicians are particularly appropriate because many physicians are uninformed about dementia ${ }^{10,11}$ and unaware of dementia services in their communities. ${ }^{12}$
From prior experience with the COEP, ${ }^{3}$ however, project staff were well aware of the challenges of involving physicians in community-based programs.

To address these challenges, advisory boards selected a variety of strategies to involve physicians in their educational outreach activities. For example, physicians were recruited as program advisors in Grand Rapids. Although they did not attend advisory board meetings, this group of physicians provided feedback on written materials by mail. In Bay City, physician office staff were trained to disseminate information about dementia to their employers. Results for the few office staff who participated were disappointing, with virtually no transfer of information to the physician. In Bay City and Saginaw, presentations about dementia were offered in a lecture format as part of Grand Rounds at local hospitals. In both sites, attendance was very low. In Grand Rapids, however, a survey of the educational needs of local physicians, using a county wide directory, provided useful information for targeting educational outreach activities and a database for disseminating educational materials. In addition, such surveys can promote awareness of local efforts in dementia education. Collaborating with staff of home health agencies to distribute educational materials to physician offices offers a promising avenue for reaching primary care physicians. Advisory board members and COEP staff have agreed to continue this activity beyond the official intervention period.

Several components of the COEP process may facilitate sustainable change in each of the four intervention sites. For example, the new or strengthened interactions among members of the advisory boards serve as the foundation for local efforts to improve dementia services. The monthly meetings of the advisory boards created ongoing opportunities for service providers to meet and exchange information and were particularly effective at strengthening relationships with local chapters of the Alzheimer's Association and home health agencies. In Saginaw, Kalamazoo, and Grand Rapids, advisory board members included an educational component as part of their monthly meetings to facilitate the exchange of current information about dementia.

A number of the activities implemented as part of the COEP can be maintained after the intervention period, including the train-the-trainer and caregiver education program as well as the Speaker's Bureau. The success of the Speaker's Bureau will be enhanced by the recruitment of a coordinator to solicit engagements, recruit and train new speakers, and organize training updates and the dissemination of materials. In addition, several of the COEP activities resulted in the transfer of new skills to community members, including public speaking, advocacy, and program planning. 
The goal of many traditional dementia education programs is to disseminate basic information about Alzheimer's disease and related disorders to narrowly defined target audiences (e.g., physicians, staff of longterm care facilities). Although such programs can reach large numbers of health professionals, community involvement is generally limited and the long term impact of such programs is rarely determined. In contrast, a community assessment was conducted in each COEP intervention site to inform an educational program that was tailored to local needs and resources. COEP staff and community participants collaborated in all aspects of program planning, implementation, and evaluation. Although this approach is time consuming and requires considerable personnel and volunteer effort, it has the potential to have a sustainable impact by strengthening the capacity of communities to respond appropriately to people with dementia and their families (for an in-depth discussion of community capacity, see Goodman et $\left.a l,{ }^{13}\right)$. In Bay City, for example, the resource center continues to expand and serve the needs of the community. Members of the Speaker's Bureau continue to schedule presentations and advocate for new services. The advisory board in Saginaw remains active and has planned additional outreach activities since the departure of the COEP. The advisory boards in Kalamazoo and Grand Rapids also continue to meet, although it is too early to determine the extent to which additional activities will be planned and implemented in these sites.

\section{Acknowledgments}

The COEP is a collaborative effort of the Michigan Alzheimer's Disease Research Center, the Michigan Public Health Institute, and the Alzheimer's Association in Michigan. The authors thank the members of the advisory boards in each site; the Executive Directors, Michigan Council and staff of the local chapters of the Alzheimer's Association in Michigan; colleagues at the Michigan Department of Community Health; and Norman Foster, Sid Gilman, and Benjamin Shaw for their contributions to the project. This research was supported by grants from the U.S. Public Health Service (NIH NIA \#P50AG08671 to the Michigan Alzheimer's Disease Research Center and
\#R25-AG11219 to the Michigan Public Health Institute) and from the Health Resources and Services Administration (CSH 000148-01-0) to the Michigan Department of Community Health.

\section{References}

1. Connell CM, Kole SL, Benedict CJ, et al: Increasing coordination of the dementia service delivery network: Planning for the Community Outreach Education Program. The Gerontologist. 1994; 34: 700-706.

2. Connell CM, Kole SL, Avey H, et al: Attitudes about Alzheimer's disease and the dementia service delivery network among family caregivers and service providers in rural Michigan. American Journal of Alzheimer's Disease. 1996; May/June: 15-25.

3. Connell CM, Kole SL: Increasing community capacity to respond to dementing illnesses: Process evaluation of the Community Outreach Education Program. Journal of Applied Gerontology. (In press).

4. Minkler M: Ten commitments for community health education. Health Education Research: Theory and Practice. 1994; 9: 527-534. 5. Israel BA, Cummings KM, Dignan MB, et al: Evaluation of health education programs: Current assessment and future directions. Health Education Quarterly. 1995; 22: 366-391.

6. Butterfoss FD, Goodman RM, Wandersman A: Community coalitions for prevention and health promotion: Factors predicting satisfaction, participation, and planning. Health Education Quarterly. 1996; 23: 65-79.

7. Delgado M: Aging research and the Puerto Rican community: The use of an elder advisory committee of intended respondents. The Gerontologist. 1996; 36: 406-408.

8. Stolee P, Kessler L, Le Clair JK: A community development and outreach program in geriatric mental health: Four years' experience. Journal of the American Geriatrics Society. 1996; 44: 314-320.

9. Simons-Morton BG, Green WA, Gottlieb N: Health education and health promotion (2nd edition). Prospect Heights, IL: Waveland. 1995.

10. Fortinsky RH, Leighton A, Wasson JH: Primary care physician's diagnostic, management and referral practices for older persons and families affected by dementia. Research on Aging. 1995; 17: 124148.

11. Rubin SM, Glasser JL, Werckle MA: The examination of physician's awareness of dementing disorders. Journal of the American Geriatrics Society. 1987; 353: 1051-1058.

12. Haley WE, Clair JM, Saulsberry K: Family caregiver satisfaction with medical care of their demented relatives. The Gerontologist. 1993; 32: 219-226.

13. Goodman RM, Speers MA, McLeroy K, et al: Identifying and defining the dimensions of a community capacity to provide a basis of measurement. Health Education and Behavior. 1998; 25: 258-278. 